\title{
Game-Theoretic Resource Allocation Algorithms for Device-to-Device Communications in Fifth Generation Cellular Networks: A Review
}

\author{
Emoghene Ogidiaka ${ }^{1}$, Francisca Nonyelum Ogwueleka $^{2}$ and Martins Ekata Irhebhude ${ }^{3}$ \\ ${ }^{1,2,3}$ Department of Computer Science, Nigerian Defence Academy, Kaduna, Nigeria \\ Email: 1'ogidiaka.emoghene@yahoo.com; ${ }^{2}$ ogwuelekafn@gmail.com; ${ }^{3}$ mirhebhude@nda.edu.ng \\ *. Ex: Corresponding author- Emoghene Ogidiaka, ogidiaka.emoghene@yahoo.com
}

Received: 14 December 2020; Accepted: 20 January 2021; Published: 08 February 2021

\begin{abstract}
Game-theoretic resource allocation algorithms are essential to managing the interference that Device-toDevice (D2D) and cellular transmissions could generate to each other in cellular networks since game-theoretic solutions are naturally autonomous and robust. In this paper, we present a survey on D2D communication in cellular networks with respect to the performance of the existing and accessible game-theoretic resource allocation algorithms published in 2013-2019. Each of the game-theoretic resource allocation algorithms with its properties such as utility, complexity, fairness, overhead cost, and convergence rate are reviewed and compared. The survey proved that gametheoretic solutions could be a viable strategy for practical implementation in 5G networks as each of the reviewed scheme attempts to optimize one or various essential performance metrics in the system. Finally, the paper recommends that serious efforts should be made by standardization bodies in incorporating game-theoretic strategy in D2D-enabled $5 \mathrm{G}$ networks while considering it as a road map for reliable and resource-efficient solutions in future cellular networks.
\end{abstract}

Index Terms: D2D communication, Resource allocation, Interference management, 5G networks, Game theory.

\section{Introduction}

The fifth-generation $(5 \mathrm{G})$ of cellular communication is the current generation networks. It is the result of an aggregation of various technologies such as heterogeneous network (HetNet), millimeter wave (mmWave) communication, cognitive radio networks (CRNs), visible light communication (VLC), massive multiple-input multiple-output (mMIMO), and device-to-device (D2D) communication [1,2]. With D2D communication as a component of the $5 \mathrm{G}$ architecture, the mmWave spectrum in $5 \mathrm{G}$ could be implemented to establish short-range D2D links among user equipment (UEs). Also, since mmWave suffers low multi-user interference, several mmWave D2D links could work simultaneously, thus enhancing network capacity. Further, secondary devices in a CRN could use D2D communication to prevent interference to primary devices. D2D communication complements HetNets and mMIMOenabled base stations (BSs) in enhancing spectral efficiency (SE) and data rates [2, 3].

D2D communication in cellular networks refers to the direct communication between two mobile devices, without having to go through the network infrastructure of access points or BS [4, 5, 3]. It is written to act as a public safety network feature in Release 12 of the Third Generation Partnership Project (3GPP) [6]. 3GPP Release 13 enables UE-tonetwork relay while 3GPP Release 14 includes support for vehicular communication based on D2D technology [7, 6]. Enhanced support for vehicle-to-everything (eV2X) services in 3GPP Release 15 will incorporate safety-related V2X use cases. Presently, 3GPP is considering and designing systems focusing on maritime communications for Release 16 and beyond to accommodate the needs of potential maritime users [6].

In general, access to the spectrum in D2D communication enabled cellular networks could be achieved by two means: Overlay spectrum mode or underlay spectrum mode [8,9]. In the overlay mode, D2D transmitters could only access the spectrum which is not used by nearby cellular devices. This approach cannot enhance spectrum utilization since the D2D pairs use different resources with cellular users (CUs). On the contrary, D2D transmitters in underlay mode could transmit on all channels subject to acceptable interference to the cellular devices. This method has the merits of not interfering with the spectrum of cellular communication but also improving the reuse gain in $\mathrm{D} 2 \mathrm{D}$ communication [9].

However, D2D technology introduces new problems; because concerning cellular communication scenarios, the system is expected to handle new interference cases, especially when it works as an underlay [5, 3]. Interference in a communication system is defined as the alteration of a signal generated from some source created by signals originating 
from other objects on its way to the destination [10]. Consequently, to fulfill the opportunities of D2D communications and handle the intra-cell and inter-cell interference, the research community has suggested several important gametheoretic resource management algorithms that can be deployed [11,2].

Game theory (GT) is a field of applied mathematics that investigates how rational players, faced with a pool of limited network resources, could compete among themselves to attain a stable allocation of system resources to fulfill the service characteristics for those players $[12,13]$. A basic game framework comprises of the following elements: players, actions, and utility functions [14]. The underlying motivation is that game-theoretic solutions are naturally autonomous, robust and a viable strategy for practical implementation in $5 \mathrm{G}$ networks [14].

Game-theoretic based resource allocation algorithms assign physical resources (that is, time/frequency, channels, transmit power levels, etc.) to cellular UE as well as D2D pairs either in a distributed or a centralized manner. Distributed techniques are easily scalable, support less message passing, but are not optimal. Centralized techniques are better in performance, but support extensive message passing $[15,16]$.

Since the aim of the various D2D communication in cellular networks-based resource allocation algorithms is different (e.g., enhancing the network capacity, improving the reliability, optimizing the sum transmission power, guaranteeing the quality of service, etc.), a survey is an appropriate way to understand the connections among each other. For example, [17] provided a survey of D2D technologies along with several characteristics including mode selection, device discovery, resource management, mobility management, and security. In [5], a comprehensive review of resource allocation methods in D2D communications was presented. Also, a survey of D2D communication in [7] concerning its architecture, use cases, and the primary technical issues to its deployment were provided. In [18], a survey of D2D communication in cellular networks was provided. Further, the authors presented a review of the D2D types based on the communication band of D2D transmission, namely inband mode, and outband mode [19].

Similarly, a survey on D2D communication was provided by [1], including the advantages it offers; its challenges including peer discovery, resource allocation; its integrant technologies like mmWave D2D, cognitive D2D, handover procedure in D2D, ultra-dense networks (UDNs), and its numerous use cases. In [20], an overview of the various modern techniques for interference prevention in D2D communication enabled cellular networks was provided. As provided in [21], an overview of the several cases of D2D communication in cellular networks from a deployment perspective was studied. The authors in [22] provided a survey of D2D communication. Further, the authors in [23] presented a review of D2D communication in cellular networks. A survey presented in [24] studied D2D standardization projects in 3GPP. Authors in [9] presented a review of the D2D direct and D2D local area network (LAN) communication use cases. However, none of these surveys focused on D2D communication in cellular networks concerning the performance of the existing game-theoretic resource allocation algorithms.

In this paper, we present a detailed and systematic survey on D2D communication in cellular networks concerning the performance of the existing and accessible game-theoretic resource allocation algorithms published in 2013-2019; taking into account the important algorithm properties such as utility, complexity, fairness, overhead cost, and convergence rate. The reason for this examination is to gain insights into the various game-theoretic schemes' suitability, especially in the $5 \mathrm{G}$ system.

The rest of this paper is organized as follows- Section 2.0 reviews several existing papers on game-theoretic resource allocation algorithms for D2D communications in cellular networks. The results of the review and comparison are discussed in Section 3.0 while Section 4.0 concludes the study.

\section{Literature Review}

A review within a period of 2013-2019 of all accessible game-theoretic resource allocation algorithms for D2D communication in cellular networks is the best way to unravel the connections among each other. In [25], the authors designed an auction-based resource allocation scheme to enhance the performance of mobile peer-to-peer, (that is, D2D) communications as an underlay in the downlink cellular networks. First, the study developed a reverse iterative combinatorial auction as the allocation approach to maximize the system sum-rate over the resource sharing for both D2D and cellular users. In the auction as proposed by the authors, the channel resources were viewed as a collection of resource units, which as bidders participate to obtain profit while the resources of the D2D pairs were auctioned off like goods in each auction cycle. The results of the simulation showed that the auction scheme led to a decent performance on the system sum-rate, converged fast, and provided high SE which has lower complexity when compared to the exhaustive search allocation algorithm.

In [14], the authors developed a game-theoretic resource allocation algorithm for intercell D2D communications in cellular networks. By adopting a repeated game framework, the authors modeled the selfish behaviour of the BS/UE and obtained the equilibrium. Further, the study concentrated on the unknown channel quality (UCQ) issue which existed specifically in D2D communication. Meanwhile, a contract-based approach with the linear search technique was suggested to solve the UCQ issue by optimizing the gain of the BS. The computer simulation result showed that the developed scheme improved system efficiency and performance, including sum rate and sum rate gain.

Authors in [8] proposed a Stackelberg game model for joint power control, spectrum sharing, and scheduling of D2D communication in heterogeneous macrocell/femtocell systems. In the study, the authors developed a utility 
function while prices for sharing the spectrum resource and proper transmit power of D2D transmitters were adjusted to optimize the utility obtained by BSs and D2D pairs. The authors analysed the optimal scheme for each participator (that is, D2D pairs/femtocell users/macrocell users), obtained the results for equilibrium state, and suggested a technique to assign resources to schedule D2D UE, where the fairness of the network and interference management were investigated. Computer simulation results showed that the developed technique could lead to an increase in transmit rate performance for both the D2D UE and the cellular.

As suggested in [26], a two-stage Stackelberg game based distributed energy-aware resource allocation algorithm for D2D communications underlay cellular networks was proposed. In the study, cellular UEs were the game leaders who charged D2D pairs for sharing link resources and D2D pairs were the game followers who purchase link resources from cellular UEs. Also, the authors modeled the utility functions as the weighted sum of accountable transmission rate, interference revenue (or cost), and power consumption. The study analysed and ascertained through computer simulations the correlation among utility functions, power consumption, interference price, and weight coefficients.

Authors in [27] proposed a coalitional game for resource allocation in the D2D uplink underlaying cellular networks. Based on the study, the overall network performance of the sum rate was formulated by a utility function in a way that all the D2D pairs and cellular devices aim to optimize. The results showed that the algorithm converges to the optimal system solution. Further, the simulation results revealed the effectiveness and fairness of the developed scheme when compared with other schemes.

Authors in [16] adopted the Stackelberg game model to develop a distributed resource allocation algorithm for joint channel reuse and power allocation challenge for D2D communications underlay a cellular network to optimize the sum data rates of D2D users while fulfilling each CU's data rate requirement. Additionally, the authors presented a centralized resource allocation mechanism through the convex approximation technique, which served as the basis for the system performance. Also, the overhead (signaling) was compared between the decentralized and centralized techniques. The numerical results showed that the distributed Stackelberg technique was efficient for the resource allocation, converge to the unique NE, and can shield the CUs with few signaling overheads.

In [28], the authors devised a combinatorial auction-based energy-efficient resource allocation algorithm for D2D underlay cellular networks. The study analysed the convergence, computational complexity, fairness, and the signaling overhead of the algorithm. The developed technique converged in finite rounds. Simulation results indicated that the developed technique achieved better performances when compared to the greedy heuristic and the fixed power schemes concerning the expected data, lifetime, and data rate. Lastly, the authors observed that by using the developed resource allocation scheme, D2D communication underlaying cellular networks could improve the system's energy efficiency when benchmarked with the traditional cellular system.

According to authors in [29], an auction-based distributed technique to address the allocation challenge for D2D communication networks was suggested. They formulated the resource allocation challenge to improve the SE and the system data rate while factoring the co-channel interference from D2D users' use of shared resources with cellular users. The computer simulation results showed that the auction-based scheme provided close to optimal results with low complexity, shared the resources fairly, and converged fast.

Authors in [3] investigated the resource allocation for D2D underlaying downlink communications in MIMO HetNets. In this study, the authors developed an effective resource allocation algorithm based on a better response dynamic for a feasible suboptimal result. The results indicated via simulations that the developed approach was quite effective and efficient, and can attain better sum-rate performance efficiency with negligible computation complexity when benchmarked against the exhaustive searching algorithm.

In [30], the authors developed a Nash bargaining GT-based resource allocation algorithm in D2D communications underlay cellular system to optimize the utility of cellular devices as well as the throughput of each D2D pair. The authors decomposed the challenge of resource allocation into channel assignment and power allocation for tractability. The results showed the superiority of the developed scheme on fairness and adjustment concerning the transmission rate performance

Authors in [31] studied auction game-based distributed resource allocation for D2D communication networks. The authors formulated the resource allocation challenge to improve the SE and the system data rate while introducing a low complexity distributed method to resolve the allocation challenge. Also, the study considered the co-channel interference when the D2D users reuse the same link resources with cellular users. Computer simulation results showed that the auction-based scheme provided close to optimal results with minimum efficiency and had low complexity.

In [32], the authors proposed a Stackelberg game-based resource allocation algorithm to jointly assign power and resources for D2D communications in a cellular network. As explained by the authors, the eNB and D2D UE make up a seller-to-buyer pair to enhance the sum throughput. In the framework, the eNB was considered as the seller and D2D UE was the buyer. Simulation results proved the effectiveness and efficiency of the developed scheme when compared with traditional methods

As suggested in [33], a distributed overlapping coalition formation game (OCFG) based resource allocation algorithm for D2D underlay cellular networks was developed. In the game, every D2D pair was permitted to join multiple coalitions at the same time to enhance the SE and optimize the system utility. Simulation results showed that 
the developed OCFG based technique outperforms the conventional techniques and provides a similar outcome to the optimal technique but with lower computational but realization complexity.

In [34], the authors studied resource allocation for an underlay D2D cellular network based on a hybrid model incorporating GT. The study designed a resource allocation framework using a learning model in terms of the Markov approximation in which they had developed a problem specific Markov chain that converged close to an optimal result. Simulation results showed that the developed model convergence in probability, showed reasonable computational complexity, achieved interference prevention, and closely approached the maximal result.

Authors in [35] suggested a GT-based resource allocation for multicell D2D communications underlaying 5G networks to maximize the system sum rate. In the study, the authors first developed a static game framework, and then expanded it to a repeated one. The study analyzed the developed scheme by proving its optimality stability.

Authors in [2] devised a coalitional game-based resource allocation for D2D communications underlay heterogeneous cellular networks to optimize the system sum rate. The study theoretically proved that the proposed game converged to a Nash-stable equilibrium and reached the close optimal result with a speedy convergence rate. The result from computer simulations demonstrated the superior performance of the algorithm in terms of the system sum-rate in comparison with other practical techniques as reviewed by the authors.

Wang et al (2018) investigated game-theoretic social-aware resource allocation for D2D communications underlaying cellular networks [36]. In the resource allocation scheme, the authors quantified both the rate enhancement brought by the social relationships between mobile devices and the rate influence from the power interference created by the D2D transmitter to cellular devices. Further, the utility function maximization game was developed to maximize the total transmission rate performance of the system. Also, the authors discussed the Nash Equilibrium of the developed utility function maximization game from a theoretical point of view and suggested a utility priority searching scheme based resource allocation algorithm. Computer simulation results indicated that the developed technique attained better performance in comparison with the other two selected schemes reviewed in the study.

In [37], the authors developed a Stackelberg game-based resource allocation algorithm for D2D communications. The proposed model aims to coordinate intra-cell interference and assign resources within the network effectively while improving D2D user quality of experience (QoE). The results, when compared to the traditional schemes, showed that the developed scheme converges to a Stackelberg Equilibrium, reduces transmit power per D2D pair while providing better social welfare and QoE across all D2D followers.

Lastly, the authors in [38] investigated the coalition game-based approach for resource allocation and transmit power control in D2D communication to maximize the system sum rate. In the study, the resource allocation challenge was developed as a single utility function for multiple D2D pairs and cellular devices in underlaying cellular networks. The results obtained through numerical simulation showed that the developed algorithm greatly enhances the system sum-rate along with reduced power consumption.

Table 1 shows a summary of the comparison of the properties of each of the accessible game-theoretic resource allocation algorithms of D2D communication in the cellular network reviewed for the study. It takes into account the important algorithm properties such as the utility, complexity, fairness, overhead cost, and convergence rate. The utility is the sum of the various optimization objectives considered by an algorithm in terms of system performance. The possible values for this attribute can be "high", "low", or "medium". The complexity is closely linked to the number of iterations, merge-and-split operations. This attribute value can be "high", "low", or "medium". Overhead is signalling which contains additional information to improve the performance of the cellular networks (e.g., information exchange over control channels). The possible values for this attribute can be "high", "low", or "medium". Fairness determines whether D2D or cellular users are getting an equal amount of system resources. The values for this attribute are "excellent", "good", or "poor". Lastly, the convergence rate describes how fast an algorithm converges to the optimal system solution. The possible values for this attribute can be "fast" or "slow".

Table 1. Comparison of accessible game-theoretic resource allocation algorithms for D2D communication in cellular networks (2013-2019)

\begin{tabular}{|l|l|l|l|l|l|l|}
\hline S/N & Literature & Utility & Complexity & Fairness & Overhead Cost & Convergence Rate \\
\cline { 3 - 6 } & {$[25]$} & $\begin{array}{l}\text { Low (System sum } \\
\text { rate) }\end{array}$ & Low & Not Applicable & Medium \\
\hline 1 & {$[14]$} & $\begin{array}{l}\text { Low (System sum } \\
\text { rate) }\end{array}$ & Not Applicable & Not Applicable & Not Applicable & Not Applicable \\
\hline 3 & {$[8]$} & $\begin{array}{l}\text { Low (Sum } \\
\text { transmission rate) }\end{array}$ & Low & Excellent & Not Applicable & Fast \\
\hline 4 & {$[26]$} & $\begin{array}{l}\text { Medium (System } \\
\text { transmission rate, } \\
\text { power consumption, } \\
\text { and interference } \\
\text { revenue) }\end{array}$ & Lot Applicable & Not Applicable & Not Applicable & Not Applicable \\
\hline 5 & {$[27]$} & {$[16]$} & $\begin{array}{l}\text { Low (Sum rate) } \\
\text { rates) (Sum data }\end{array}$ & Not Applicable & Not Applicable & Medium \\
\hline 6 & &
\end{tabular}




\begin{tabular}{|c|c|c|c|c|c|c|}
\hline 7 & [28] & $\begin{array}{ll}\text { Low } & \text { (Energy } \\
\text { efficiency) }\end{array}$ & Medium & Excellent & Low & Fast \\
\hline 8 & [29] & $\begin{array}{l}\text { Medium (SE and } \\
\text { the system data rate) }\end{array}$ & Low & Excellent & Not Applicable & Fast \\
\hline 9 & [3] & Low (Sum rate) & Low & Not Applicable & Not Applicable & Fast \\
\hline 10 & [30] & Low (Sum rate) & Low & Excellent & Not Applicable & Not Applicable \\
\hline 11 & [31] & $\begin{array}{l}\text { Medium (SE and } \\
\text { system data rate) }\end{array}$ & Low & Excellent & Low & Fast \\
\hline 12 & [32] & Low (Sum rate) & Not Applicable & Not Applicable & Not Applicable & Fast \\
\hline 13 & [33] & $\begin{array}{ll}\begin{array}{l}\text { Low } \\
\text { throughput })\end{array} & \text { (Sum } \\
\end{array}$ & Low & Not Applicable & Not Applicable & Fast \\
\hline 14 & [34] & Low (Sum rate) & Low & Medium & Not Applicable & Fast \\
\hline 15 & [35] & Low (Sum rate) & Not Applicable & Not Applicable & Not Applicable & Not Applicable \\
\hline 16 & [2] & $\begin{array}{l}\text { Low (System sum- } \\
\text { rate) }\end{array}$ & Low & Not Applicable & Not Applicable & Fast \\
\hline 17 & [36] & $\begin{array}{l}\text { Low (System sum- } \\
\text { rate) }\end{array}$ & Not Applicable & Not Applicable & Not Applicable & Fast \\
\hline 18 & [37] & $\begin{array}{l}\text { High (Power, data } \\
\text { rate, QoE) }\end{array}$ & Not Applicable & Not Applicable & Not Applicable & Fast \\
\hline 19 & {$[38]$} & $\begin{array}{l}\text { Medium (Sum rate } \\
\text { and power } \\
\text { consumption) }\end{array}$ & Not Applicable & Not Applicable & Not Applicable & Fast \\
\hline
\end{tabular}

\section{Results and Discussion}

The results of the review showed that all surveyed papers' game-theoretic resource allocation algorithms achieved low network interference with Stackelberg, coalition, bargaining, and auction as the preferred game models adopted. Interestingly, all surveyed papers considered the D2D underlay mode with about $78.9 \%$ of the surveyed algorithms focused on the uplink frame of the network, within a single cell deployment. This could be to the fact that D2D UEs suffer serious interference from the BS in the downlink, due to the large transmit power of BS compared to UEs.

Also, it was observed that about $73.6 \%$ of the surveyed schemes have low utility as they focused on a single optimization goal. This is not surprising because of the difficulty in striking a balance among multiple conflicting objective functions. However, guaranteeing high utility with multiple optimization objectives ensures end-user satisfaction, and it is becoming a vital quality of measure in the $5 \mathrm{G}$ cellular system in meeting the demands of users. Nevertheless, it is worth pointing out that all surveyed schemes in this study have system sum-rate as an optimization objective. This may be due to its importance in today's $5 \mathrm{G}$ systems.

Similarly, the convergence rate and the amount of complexity of most schemes proposed were fast and very limited. As a result, it shows a reasonable convergence rate and computational complexity for practical implementation in the current and future cellular networks. Further, 26.3\% of the proposed game-theoretic resource allocation algorithms achieved a high-performance level in terms of fairness to D2D users. This indicates that D2D users are no longer treated as secondary users of the cellular network; hence their service quality should not be sacrificed because of CUs users in the implementation of practical design for $5 \mathrm{G}$ networks.

Additionally, it was observed that about $15.8 \%$ of the surveyed studies consider the overhead cost which is still limited; hence, it would not affect the efficiency of these schemes when applied in 5G networks. Also, we noticed that all parameters of utility, complexity, fairness, signalling overhead cost, and convergence were rarely considered as a whole by the surveyed schemes. This is evident as about $10.5 \%$ of the surveyed schemes including the studies of [28] and [31] were efficient in achieving optimal solutions regarding all performance metrics considered. Given these observations, we suggest that serious efforts should be made in this direction in future research studies.

\section{Conclusion}

This paper has presented a survey on D2D communication in $5 \mathrm{G}$ cellular systems concerning the performance of the existing and accessible game-theoretic resource allocation techniques published in 2013-2019. Game-theoretic frameworks help develop resource allocation schemes to achieve steady and effective results by encouraging D2D users to efficiently share the approved spectrum of cellular users. The performance of each game-theoretic scheme was analyzed taking into account the important algorithm properties such as the utility, complexity, fairness, overhead cost, and convergence rate for $5 \mathrm{G}$ networks. The survey showed that each of them tries to optimize one or various essential performance metrics in the system. Also, all proposed schemes from the surveyed papers considered the D2D underlay mode, have system sum-rate as an optimization objective, and achieved low network interference. These results tend to suggest that game-theoretic solutions could be a viable strategy to the challenge of resource allocation in controlling the interference in cellular networks, especially for the $5 \mathrm{G}$ network.

However, based on the various game-theoretic resource allocation algorithms surveyed in this study, we have observed that [28] and [31] have provided the best performance. The utility, complexity, fairness, overhead cost, and 
convergence rate have been maximized in these algorithms, which are desirable from the user perspective as well as the service provider. Given these observations, and with the deployment of the 5G network still under progress, we recommend that serious efforts should be made by standardization bodies in incorporating game-theoretic strategy in D2D-enabled 5G networks. This will help improve its design, fast-track its current deployment, contribute to its longrun growth while providing a road map for reliable and resource-efficient solutions in future cellular networks. Finally, since the resource which can be used in cellular communication is very limited, this paper has shown that taking full and efficient use of it in the communication system via game-theoretic-interference-aware strategy will become more and more important in the future.

\section{References}

[1] Gandotra, P., and Jha, R. K. (2016). Device-to-Device Communication in Cellular Networks: A Survey. Journal of Network and Computer Applications, Vol. 71, No.1, pp. 99-117.

[2] Chen, Y., Ai, B., Niu, Y., Guan, K., and Han, Z. (2018). Resource Allocation for Device-to-Device Communications Underlaying Heterogeneous Cellular Networks using Coalitional games. IEEE Transactions on Wireless Communications, Vol. 17, No.6, pp. 4163-4176.

[3] Zhong, W., Fang, B., and Qian, Z. (2015). Game-Theoretic Resource Allocation for D2D MIMO Heterogeneous Networks. Wireless Personal Communications, Vol.84, No.1, pp. 273-285.

[4] Feng, W., Wang, Y., and Yang, L. (2013). Resource Allocation Method of Device-to-Device Communication. Communications and Network, Vol. 5, No. 3, pp. 338.

[5] Yu, S., Ejaz, W., Guan, L. and Anpalagan A (2017). Resource Allocation Schemes in D2D Communications: Overview, Classification, and Challenges Wireless Personal Communications, Vol. 96, No. 1, pp. 303-322

[6] Höyhtyä, M., Apilo, O., and Lasanen, M. (2018). Review of Latest Advances in 3GPP Standardization: D2D communication in 5G Systems and its Energy Consumption Models. Future Internet, Vol 10, No 1, pp. 1-18.

[7] Kar, U. N., and Sanyal, D. K. (2017). An Overview of Device-to-Device Communication in Cellular Networks. ICT Express. KIIT University, Bhubaneswar, India, pp.1-7.

[8] He, Y., Wang, F., and Wu, J. (2014). Resource Management for Device-to-Device Communications in Heterogeneous Networks using Stackelberg Game. International Journal of Antennas and Propagation, Vol. 1, No 1, pp. 1-10.

[9] Song, L., Niyato, D., Han, Z., and Hossain, E. (2014). Game-Theoretic Resource Allocation Methods for Device-to-Device Communication. IEEE Wireless Communications, Vol 21, No.3, pp.136-144.

[10] Islam, M. (2016). Radio Resource Allocation for Device-to-Device Communications Underlaying Cellular Networks, (Doctoral dissertation), Queen's University, Kingston, Ontario, Canada, pp. 1-153.

[11] Oulaourf, S., Haidine, A., Aqqal, A., and Ouahmane, H. (2017). Review on Radio Resource Allocation Optimization in LTE/LTE-Advanced using Game Theory. International Journal of Communication Networks and Information Security (IJCNIS), Vol. 9, No.1, pp.117-153.

[12] MacKenzie, A. B., and DaSilva, L. A. (2006). Game Theory for Wireless Engineers. Synthesis Lectures on Communications, Vol.1, No 1, pp. 1-86.

[13] Moura, J., and Hutchison, D. (2017). Survey of Game Theory and Future Trends with Application to Emerging Wireless Data Communication Networks. CoRR., pp 1-50. [Online document] Available: Researchgate. net/publication, 315764798. [Accessed: March, 2019].

[14] Huang, J., Yin, Y., Zhao, Y., Duan, Q., Wang, W., and Yu, S. (2014). A Game-Theoretic Resource Allocation Approach for Intercell Device-to-Device Communications in Cellular Networks. IEEE Transactions on Emerging Topics in Computing, Vol.4, No.4, pp.475-486.

[15] Alamouti, S. M., and Sharafat, A. R. (2016). Resource Allocation for Device-to-Device Communications in Multi-Cell LTEAdvanced Wireless Networks with C-RAN Architecture. IEEE ITU Kaleidoscope: ICTs for a Sustainable World (ITU WT), Bangkok, Thailand, pp. 1-8.

[16] Yin, R., Zhong, C., Yu, G., Zhang, Z., Wong, K. K., and Chen, X. (2015). Joint Spectrum and Power Allocation for D2D communications Underlaying cellular networks. IEEE Transactions on Vehicular Technology, Vol. 65, No 4, pp. 2182-2195.

[17] Jameel, F., Hamid, Z., Jabeen, F., Zeadally, S., and Javed, M. A. (2018). A Survey of Device-to-Device Communications: Research Issues and Challenges. IEEE Communications Surveys \& Tutorials, Vol. 20, No.3, pp. 2133-2168

[18] Rathi, R., and Gupta, N. (2017). A Review of D2D Communication with Game-Theoretic Resource Allocation Models. IEEE 2017 International Conference on Next Generation Computing and Information Systems (ICNGCIS), Jammu, India, pp. 142146.

[19] Hicham, M., Abghour, N., and Ouzzif, M. (2016). Device-to-device (D2D) communication under LTE-advanced networks. International Journal of Wireless \& Mobile Networks (IJWMN) Vol, 8. No 1, pp. 1-12

[20] Noura, M., and Nordin, R. (2016). A Survey on Interference Management for Device-to-Device (D2D) Communication and its Challenges in 5G Networks. Journal of Network and Computer Applications, Vol.71, No.1, pp.130-150.

[21] Sakr, A. H., Tabassum, H., Hossain, E., and Kim, D. I. (2015). Cognitive Spectrum Access in Device-to-Device-Enabled Cellular Networks. IEEE Communications Magazine, Vol 53, No. 7, pp.126-133.

[22] Alkurd R, Shubair M R. and Abualhaol I (2014). Survey on Device-to-Device Communications: Challenges and Design Issues pp. $1-5$

[23] Asadi, A., Wang, Q., and Mancuso, V. (2014). A Survey on Device-to-Device Communication in Cellular Networks. IEEE Communications Surveys \& Tutorials, Vol. 16, No. 4, pp. 1801-1819.

[24] Lin, X., Andrews, J. G., Ghosh, A., and Ratasuk, R. (2014). An Overview of 3GPP Device-to-Device Proximity Services. IEEE Communications Magazine, Vol.52, No.4, pp. 40-48. 
[25] Xu, C., Song, L., Han, Z., Zhao, Q., Wang, X., Cheng, X., and Jiao, B. (2013). Efficiency Resource Allocation for Device-toDevice Underlay Communication Systems: A Reverse Iterative Combinatorial Auction Based Approach. IEEE Journal on Selected Areas in Communications, Vol. 31, No 9, pp. 348-358.

[26] Zhou, Z., Dong, M., Ota, K., Gu, B., and Sato, T. (2014). Stackelberg-Game Based Distributed Energy-Aware Resource Allocation in Device-to-Device Communications. IEEE International Conference on Communication Systems, Macau, China, pp. 11-15.

[27] Li, Y., Jin, D., Yuan, J., and Han, Z. (2014). Coalitional Games for Resource Allocation in the Device-to-Device Uplink Underlaying Cellular Networks. IEEE Transactions on Wireless Communications, Vol. 13, No 7, pp. 3965-3977.

[28] Wang, F., Xu, C., Song, L., and Han, Z. (2015). Energy-Efficient Resource Allocation for Device-to-Device Underlay Communication. IEEE Transactions on Wireless Communications, Vol. 14, No 4, pp. 2082-2092.

[29] Almofari N, Kishk S, and Zaki W. F (2015) Distributed Resource Allocation for D2D Communication Networks Based in Game Theory International Journal of Innovative Research in Electronics and Communications (IJIREC) Vol. 3, No 4, pp. 3543

[30] Liu, T., and Wang, G. (2015). Resource Allocation for Device-to-Device Communications as an Underlay Using Nash Bargaining Game Theory. International Conference on Information and Communication Technology Convergence (ICTC), pp. 366-371.

[31] Zaki F. W., Kishk S., and Almofari N. H. (2016) Distributed Resource Allocation for D2DCommunication Networks Based in Game Theory International Journal of Innovative Research in Electronics and Communications (IJIREC) Vol. 3, No.4, pp. $35-43$

[32] Xu S. and Xia C. (2016), Resource Allocation for Device-to-Device Communications in LTE-A Network: A Stackelberg Game Theory Approach,pp.

[33] Xu, S., Xia, C., and Kwak, K. S. (2016). Overlapping Coalition Formation Games Based Interference Coordination for D2D underlaying LTE-A networks. AEU-International Journal of Electronics and Communications, Vol. 70, No. 2, pp. $204-209$.

[34] Kazmi, S. A., Tran, N. H., Saad, W., Han, Z., Ho, T. M., Oo, T. Z., and Hong, C. S. (2017). Mode Selection and Resource Allocation in Device-to-Device Communications: A Matching Game Approach. IEEE Transactions on Mobile Computing, Vol. 16, No. 11, pp. 3126-3141.

[35] Huang, J., Xing, C. C., Qian, Y., and Haas, Z. J. (2017). Resource allocation for Multicell Device-to-Device Communications Underlaying 5G Networks: A Game-Theoretic Mechanism with Incomplete Information. IEEE Transactions on Vehicular Technology, Vol. 67, No. 3, pp. 2557-2570.

[36] Wang, L., Li, C., Zhang, Y., and Gui, G. (2018). Game-Theoretic Social-Aware Resource Allocation for Device-to-Device Communications Underlaying Cellular Network. Wireless Communications and Mobile Computing, pp?

[37] Sawyer, N., and Smith, D. B. (2018). Flexible Resource Allocation in Device-to-Device Communications Using Stackelberg Game Theory. IEEE Transactions on Communications, Vol. 67, No.1, pp. 653-667.

[38] Sharma, S., and Singh, B. (2019). Coalition Game-Based Strategy for Resource Allocation and Transmit Power Control in D2D Communication. National Academy Science Letters, pp. 1-3.

\section{Authors' Profiles}

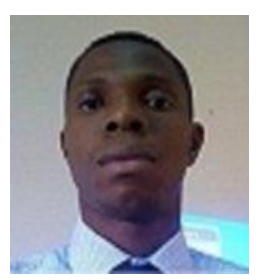

Dr. Ogidiaka Emoghene holds a BSc, MSc, and Ph.D. degrees in Computer Science. His primary research interests are in Cloud Computing, Software Programming, Internet of Things (IoT), Fifth Generation (5G), Artificial Intelligence (AI), Big Data, Computer Networks, and Cyber Security. He currently works for Teledom International Limited, Lagos, Nigeria as a Network Engineer

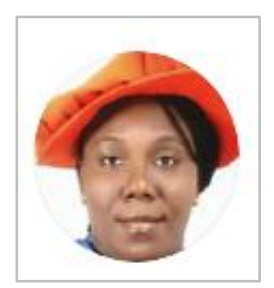

Professor Dr. Francisca Nonyelum Ogwueleka holds a B.Eng Computer Science \& Engineering, MSc Computer Science, and Ph.D. Computer Science degree. She is a professor of Computer Science with expertise in Data Mining, Computer Security and Reliability, Artificial Neural Network. She currently lectures at the Nigerian Defence Academy, Kaduna, Nigeria. 
Dr. Martins Ekata Irhebhude holds a BSc, MSc, and Ph.D. degrees in Computer Science. He currently lectures at the Nigerian Defence Academy, Kaduna, Nigeria. His research interests include: Image Processing, Object Detection, People Tracking, People Re-identification, Object Recognition, Artificial Intelligence, and Computer Vision related researches

How to cite this paper: Emoghene Ogidiaka, Francisca Nonyelum Ogwueleka, Martins Ekata Irhebhude, " Game-Theoretic Resource Allocation Algorithms for Device-to-Device Communications in Fifth Generation Cellular Networks: A Review", International Journal of Information Engineering and Electronic Business(IJIEEB), Vol.13, No.1, pp. 44-51, 2021. DOI: 10.5815/ijieeb.2021.01.05 